\title{
Tumor Necrosis in Hepatocellular Carcinoma-Unfairly Overlooked?
}

\author{
Lana Bijelic, $\mathrm{MD}^{1}$, and Emilio Ramos Rubio, $\mathrm{MD}^{2}$ \\ ${ }^{1}$ Division of Surgical Oncology, Department of Surgery, Hospital Moises Broggi, Barcelona, Spain; ${ }^{2}$ Hepatobiliary \\ Surgery, Department of Surgery, Hospital Universitario de Bellvitge, IDIBELL, CIBERehd, Barcelona, Spain
}

Hepatocellular carcinoma (HCC) is a major source of cancer-related mortality worldwide due to its high global incidence, but also the fact that we have relatively few tools to fight it with, especially at advanced stages.' The complex biology of HCC itself, closely interlinked with the underlying condition of the organ where it appears, further challenges any attempts to improve therapeutic options. Our ability to subdivide patients into prognostically relevant and well-defined subgroups by using easily accessible clinicopathological variables is essential not only for everyday clinical management decisions, but also to help better design and interpret clinical trials of various therapeutic interventions.

In this issue of the Annals, a report by Wei et al. offers a new look at tumor necrosis, a simple, easily assessed, albeit nonspecific, histopathologic variable, and its correlation with outcomes in surgically resected HCC. ${ }^{2}$ The report is based on prospectively collected data from a large multiinstitutional registry (919 patients) of high-volume hepatobiliary centers from around the world. It stands out for the authors' ability to tell their story with clarity and elegance. Not only was HCC necrosis associated with overall and recurrence-free survival in the whole cohort, as well as being more frequently present in tumors with progressively worse overall characteristics, but necrosis of $>50 \%$ was also able to "upstage" smaller and more favorable tumors (T1 HCC with $>50 \%$ necrosis had survival equivalent to a

(C) Society of Surgical Oncology 2020

First Received: 6 November 2020

Accepted: 10 November 2020;

Published Online: 22 November 2020

L. Bijelic, MD

e-mail: lana.bijelic@csi.cat
$\mathrm{T} 2$ tumor, and $\mathrm{T} 2$ tumors with $>50 \%$ necrosis became indistinguishable from $\mathrm{T} 3$ tumors in terms of survival). This cohort is the largest and most comprehensive analysis of tumor necrosis in resected HCC, which is a strength of this study compared with the few others available on the topic.

There are, however, several potential confounders to consider. Spontaneous necrosis can be seen in HCC and many other solid tumors and is often prognostic, as shown in this study as well as in numerous other examples in renal, colon, breast, and lung cancer. ${ }^{3}-5$ However, it has not been accepted as part of required pathology reporting in any of them and is, therefore, not fully standardized. In the present report, each institution followed internal protocols at the time of tumor necrosis assessment with the possibility of some differences among institutions in terms of scoring, especially related to assigning the percent of tumor necrosis. In addition, little is known about any potential influence of underlying histological changes in the tissue, such as inflammation, fibrosis, and cirrhosis of the liver, and the reliability and reproducibility of tumor necrosis scoring in HCC, or about any potential interaction between the presence of extensive necrosis and the assessment of other important histological features such as vascular invasion.

In addition to spontaneous necrosis within the tumor, necrosis can also be associated with inflammation produced in the setting of various host-related conditions or caused by cancer-directed treatments delivered prior to surgery. Although neoadjuvant therapy is not recommended routinely prior to resection of $\mathrm{HCC}$, various modalities have been studied and continue to be used, especially for downstaging in cases of borderline resectability. Transarterial chemoembolization (TACE) and transarterial radioembolization (TARE) have been the 
most studied modalities, and both are associated with tumor necrosis. The largest experiences with preoperative TACE and TARE come from China and other Eastern countries, which form part of the registry evaluated by the Wei et al. study. ${ }^{6}$ The authors do not give us detailed information about inclusion criteria in the "Methods" section, even though their "Abstract" suggests that patients used for this analysis were previously untreated, presumably including all forms of neoadjuvant therapy. However, as we think about the potential application of tumor necrosis status in the real world, we have to consider the possibility that neoadjuvant therapy would be more frequently used in patients with worse overall prognostic features, the same group where the highest incidence and extent of necrosis is observed, thus making the interpretation of the score more difficult. We also lack information regarding the hepatitis B status of the patients in this study, another variable that has been associated with worse survival and recurrence in HCC and that could influence the multivariable analysis, especially if a substantial number of patients in the cohort are from countries with high prevalence of HBV infections.

The analyzed cohort includes only patients who underwent surgical resection. However, the majority of patients with HCC will not be treated surgically because of their impaired liver function or stage of disease. It is therefore unknown whether the reported frequency and prognostic value of tumor necrosis would remain the same if an even broader set of cases, not only surgically resected ones, were analyzed. Studies based on tissue samples of all patients with HCC are challenging to carry out because pretreatment biopsy is not clinically indicated in a large percentage of patients. However, this type of assessment would help further validate the prognostic value of tumor necrosis. It would also help better understand the optimal clinical application of tumor necrosis status: as a selection tool for choice of treatment at the onset (such as the use of neoadjuvant therapy), as a marker of higher risk of systemic spread and potential usefulness of adjuvant therapy (for example, as one of the inclusion criteria or a stratification factor for clinical trials of postoperative systemic therapy), or simply as an aid when discussing prognosis with patients. A recent example of a randomized prospective trial of adjuvant liver-directed therapy with TACE teaches us that finer stratification by risk of recurrence may be key in determining the benefit (or lack thereof) of adjuvant therapy in $\mathrm{HCC}^{8}$. The recent positive results of immune checkpoint inhibitors, such as atezolizumab, in advanced HCC are likely to lead to more trials exploring similar therapeutic intervention in the adjuvant setting, and a better understanding of prognostic stratification can be useful in optimizing trial design.

Until then, we may not be ready to change clinical practice just yet but will probably pay a bit more attention to the often-overlooked mention of tumor necrosis in the pathology report of a patient with resected HCC.

DISCLOSURE No commercial interest or source of any financial or material support.

\section{REFERENCES}

1. Bray F, Ferlay J, Soerjomataram I, Siegel RL, Torre LA, Jemal A. Global cancer statistics 2018: GLOBOCAN estimates of incidence and mortality worldwide for 36 cancers in 185 countries. CA Cancer J Clin 2018; 68: 394-424.

2. Wei T, MD, PhD1, Zhang X, MD, Bagante F et al. tumor necrosis impacts prognosis of patients undergoing curative-intent hepatocellular carcinoma after curative-intent resection. Ann Surg Oncol 2020 , in press

3. Sengupta S, Lohse CM, Leibovich BC et al. Histologic coagulative tumor necrosis as a prognostic indicator of renal cell carcinoma aggressiveness. Cancer 2005; 104: 511-520.

4. Fisher ER, Land SR, Saad RS et al. Pathologic variables predictive of breast events in patients with ductal carcinoma in situ. Am J Clin Pathol 2007; 128: 86-91.

5. Pollheimer MJ, Kornprat $\mathrm{P}$, Lindtner RA et al. Tumor necrosis is a new promising prognostic factor in colorectal cancer. Hum Pathol 2010; 41: 1749-1757 (2010).

6. Qi X, Liu L, Wang et al. Hepatic resection alone versus in combination with pre and post-operative transarterial chemoembolization for the treatment of hepatocellular carcinoma: A systematic review and meta-analysis. Oncotarget 2015; 6(34): 36838-36859.

7. Zhou HB, Li QM, Zhong ZR, et al. Level of hepatitis B surface antigen might serve as a new marker to predict hepatocellular carcinoma recurrence following curative resection in patients with low viral load. Am J Cancer Res 2015; 5:756-71.

8. Hirokawa F, Komeda K, Taniguchi K et al. Is postoperative adjuvant transcatheter arterial infusion therapy effective for patients with hepatocellular carcinoma who underwent hepatectomy? A prospective randomized controlled trial. Ann Surg Oncol. 2020. https://doi.org/10.1245/s10434-020-08699-w.

9. Finn R MD, Qin S MD, Ikeda M MD et al. Atezolizumab plus bevacizumab in unresectable hepatocellular carcinoma. N Engl J Med 2020; 382:1894-905.

Publisher's Note Springer Nature remains neutral with regard to jurisdictional claims in published maps and institutional affiliations. 